\title{
Performance evaluation of SMOS sea surface salinity observations in retrieving salinity profiles
}

\author{
Jian Chen, Ren Zhang, Luhua Wang, Gongjie Wang \\ PLA Key Laboratory of Marine Environment \\ Institute of Meteorology and Oceanography, PLA University of Science and Technology \\ Nanjing, China
}

\begin{abstract}
The Soil Moisture and Ocean Salinity (SMOS) remotely sensed sea surface salinity (SSS) observations, on a global scale and with various resolutions, have been applied as inputs in three representative retrieval techniques. The purpose is to evaluate the SSS observations' performance, concerning the accuracy of the salinity (S) profiles they retrieve, the spatial scales they can effectively resolve, and the way their errors are projected at depth. The SMOS SSS errors are small in the tropics and large in the extratropics. The spatial resolution, rather than temporal one, is an important factor for the accuracy of retrieved $\mathrm{S}$. In the upper $\sim 100 \mathrm{~m}$ mixed layer, on average, the root mean square (rms) of retrieved $S$ is larger than, equivalent to, or smaller than the signal rms, with a SSS resolution of $1 / 4^{\circ}, 1 / 2^{\circ}, 1^{\circ}$, respectively, in the tropics, and always larger elsewhere. This can be explained by the SSS-S regression coefficients, which are uniformly large in the mixed layer and thus project vertically most of the surface error features. Meanwhile, the projection generally reduces the mesoscale structures in the $1 / 4^{\circ} \mathrm{SSS}$ fields. Below the mixed layer, mainly influenced by the sea level anomaly instead, the regions with high-accuracy retrieved $S$ displace to higher latitudes. The SSS error patterns disappear, except in the southern oceans where the abnormally large SSS-S regression coefficients project SSS errors down to $400 \sim 500 \mathrm{~m}$.
\end{abstract}

Index Terms-SMOS, sea surface salinity, salinity retrieval, performance evaluation, spatial resolution.

\section{INTRODUCTION}

The performance of remotely sensed sea surface salinity (SSS) observations in reconstructing global 3-D temperature (T) and salinity (S) fields has never been evaluated using data from the Soil Moisture and Ocean Salinity (SMOS) [1] or Aquarius/SAC-D missions. Among studies on the SSS measurements' role in reconstructing profiles or on the retrieval methods' sensitivity to SSS errors, the SSS measurements were obtained only through drifting/moored buoys [2], Argo floats [3], low-resolution [4] or high-resolution [5] in-situ-observation-based gridded products. It should be emphasized that a simple/ideal error analysis, e.g., adding random white noises to the surface in situ values used as input for the vertical reconstruction seems inadequate, because fully independent remotely sensed SSS data from the SMOS or Aquarius missions could be contaminated by various kind of errors, and there are still many uncertainties about their real accuracy and geographical flavors. For SMOS mission, for instance, SSS errors potentially stem from instrument observation, brightness temperature reconstruction, salinity retrieval, gridding processing, etc., and differ at different regions [6], which together complicate the practical application of SSS data. Even in the operational system newly developed within the MESCLA [5], the SSS fields used as inputs are still based on in situ observations. This work thus represents the first attempt to apply purely remotely sensed SSS observations, on a global scale and with various resolutions, in retrieval techniques and evaluates their performance.

\section{DATA}

The in situ T/S profiles are from the CORA3 dataset of the Coriolis data center. Only Argo profiles containing both T and $\mathrm{S}$ (used to calculate a dynamic height (anomaly), i.e. DH(A)) and valid up to $1000 \mathrm{~m}$ depth are selected to give a total of 631982 profiles. These observations are pre-processed according to Argo recommendations for data quality control and interpolated to standardized levels, and used as training data (during $2000 \sim 2010$ ) and test data (during 2011).

The monthly ARIVO T/S fields defined on a $1 / 2^{\circ}$ Mercator grid from 0 to $2000 \mathrm{~m}$ depth are used for comparison with the gridded $\mathrm{S}$ fields estimated from satellite inputs, and the averages over 2004 to 2010 are used as monthly climatologies.

The SMOS SSS data are obtained from the Ocean Salinity Expertise Center (CECOS) of the CNES-IFREMER Centre Aval de Traitemenent des Donnees SMOS (CATDS), at IFREMER, Plouzane (France), 2010-2012, V02. Six types of products are used: 10 days composite with $1 / 4^{\circ}, 1 / 2^{\circ}$, and $1^{\circ}$ resolution; monthly composite with $1 / 4^{\circ}, 1 / 2^{\circ}$, and $1^{\circ}$ resolution. The sea surface temperature (SST) data with daily and $1 / 4^{\circ}$ resolution are from Reynolds L4 analyses distributed by the National Climatic Data Center at NOAA. The altimeter sea level anomalies (SLA) data with daily and $1 / 4^{\circ}$ resolution are from the Ssalto/Duacs center (AVISO, 2012). The SLA fields are recalculated using the $11 \mathrm{yr}$ time mean from 2000 to 2010. The steric components of SLA are extracted using regression coefficients from an in situ/altimeter comparison study [7]. The SLA and SST maps are combined over the SSS composite period (10 days or one month) and remapped on the SSS grids (when necessary) using a bilinear interpolation. The satellite data of the year 2011 have been used. 


\section{METHODS}

\section{A. Retrieval methods}

For generalization, three representative methods are used.

Linear regression (Method I): The $\mathrm{S}$ can be directly estimated through simple or multiple linear regressions [5, 8]:

$$
\begin{gathered}
\delta S(z)=\gamma(z) \cdot \delta S L A, \\
\delta S(z)=\lambda(z) \cdot \delta S L A+\theta(z) \cdot \delta S S S,
\end{gathered}
$$

where $\delta$ denotes anomalies with respect to monthly climatologies; $\gamma$ and $\lambda / \theta$ which vary with depth, location, and time (only depth $z$ are displayed for clarity) are regression coefficients of SLA or SLA/SSS onto S.

EOF decomposition (Method II): This method begins with the empirical orthogonal function (EOF) decomposition of observed $\mathrm{S}$ profiles, and then computes a regression between the amplitudes of the EOF modes (i.e., PCs) and SLA or SLA/SSS. The univariate EOF method by Carnes et al. [9] is adopted here, despite of the more recent but intrinsically same bivariate/multivariate EOF methods [2].

Intelligent method (Method III): Several nonlinear intelligent methods, such as neural networks and genetic algorithm [3], have been applied in T/S retrieval. The selforganizing map (SOM) classification [4] using SST/SLA or SST/SLA/SSS, as a reliable retrieval technique, is adopted here.

\section{B. Evaluation scheme}

First, for each method, a global description of the statistical relationships between surface and subsurface fields is performed using historical (2000-2010) in situ observations. For Method I, II, seasonal regression coefficients are computed on each $1^{\circ}$ grid using all observations in an influence radius of $5^{\circ}$ in latitude and $10^{\circ} \sim 25^{\circ}$ in longitude to reach a minimum number of 500 profiles; for Method III, SOM maps was trained from all the data within each $15^{\circ} \times 15^{\circ}$ box (as in Fig. 3).

Then, the methods are tested using independent (2011) in situ and satellite surface values, respectively. The retrieved profiles, as well as climatology, are compared with the independent in situ profiles. The root mean square (rms) difference between in situ $\mathrm{S}$ and the ARIVO climatology, the $\mathrm{S}$ estimated from in situ surface values (e.g., Eq. 2), the $S$ from collocated satellite surface values (e.g., Eq. 2), and the $S$ from collocated satellite SST/SLA only (e.g., Eq. 1) are denoted by $\sigma_{0}, \sigma_{1}, \sigma_{2}$, and $\sigma_{3}$, respectively. Among them, $\sigma_{0}$ represents the signal rms of the $\mathrm{S}$ fields, $\sigma_{1}$ represents the errors due to the methods, while the errors due to the inaccuracy in satellite surface values can be associated to the regression coefficients in this way (e.g. in Method I):

$$
\sigma_{2}(z)-\sigma_{1}(z) \approx \lambda(z) \cdot \sigma_{S L A}+\theta(z) \cdot \sigma_{S S S}
$$

where $\sigma_{S L A}$ and $\sigma_{S S S}$ are errors in remote sensed SLA and SSS. In addition, as the $S$ used to be estimated from SST/SLA only (e.g., Eq. 1), the modification to include additional SSS information (e.g., Eq. 2) needs to be proved necessary in terms of introducing smaller errors. That is why $\sigma_{3}$ is introduced. These rms differences could be alternately expressed as percentage of signal rms: $p_{1}=\sigma_{1} / \sigma_{0}, p_{2}=\sigma_{2} / \sigma_{0}, p_{3}=\sigma_{3} / \sigma_{0}$.

\section{RESULTS}

The evaluation first concerns the tropical Pacific Ocean where the accuracy of SMOA SSS data is relatively reasonable, and begins with qualitatively looking at the salinity patterns, at the surface, namely the input fields, and at $100 \mathrm{~m}$ depth, which corresponds approximately to the base of the mixed layer in the basin (Fig. 1). At the surface, the ARIVO SSS field and SMOS $1^{\circ}$ SSS field show uniform and smooth patterns, such as the Western Pacific Salinity Front clearly denoted by the 34.6 isohaline, and the Eastern Pacific Fresh Pool off the Panama Bight. The SMOS $1 / 2^{\circ}$ SSS field show basically identical but less smooth appearance. The SMOS $1 / 4^{\circ} \mathrm{SSS}$ field, in contrast, appears strikingly "noisy", with the 34.6 isohaline, e.g., connecting to each other from the west and east, which leads to the aliasing of largescale signals. At $100 \mathrm{~m}$, except in parts of the southeastern tropical Pacific Ocean for SMOS $1 / 4^{\circ} \mathrm{S}$ field, the $\mathrm{S}$ fields estimated from all the inputs display consistently smooth patterns, despite of their discrepancies in surface structures. Additional analysis using Method II and III (not shown) came to analogous conclusions. Thus, the vertical projection generally reduces the mesoscale features. However, it remains questionable whether they are real signals or just noises. A quantitative analysis is thus required on the spatial scales that the SMOS SSS can effectively resolve.

As in Fig. 2, even the use of in situ SSS measurements reduces the rms differences of $\mathrm{S}$ by $90 \sim 100 \%$ at the surface down to $\sim 10 \%$ at depth, the use of satellite SSS measurements, owning to their large errors of $0.25 \sim 0.3 \mathrm{psu}$, can improve the $\mathrm{S}$ estimates to a very limited extent. In the upper $\sim 100 \mathrm{~m}$ mixed layer, dependent on the spatial resolutions of satellite SSS, $\sigma_{2}$ is larger than $\left(1 / 4^{\circ}\right)$, roughly equal to $\left(1 / 2^{\circ}\right)$, or smaller than $\left(1^{\circ}\right)$ $\sigma_{0}\left(\right.$ and $\left.\sigma_{3}\right)$. Between 100 and $300 \mathrm{~m}$ (Method I, II) or $250 \mathrm{~m}$

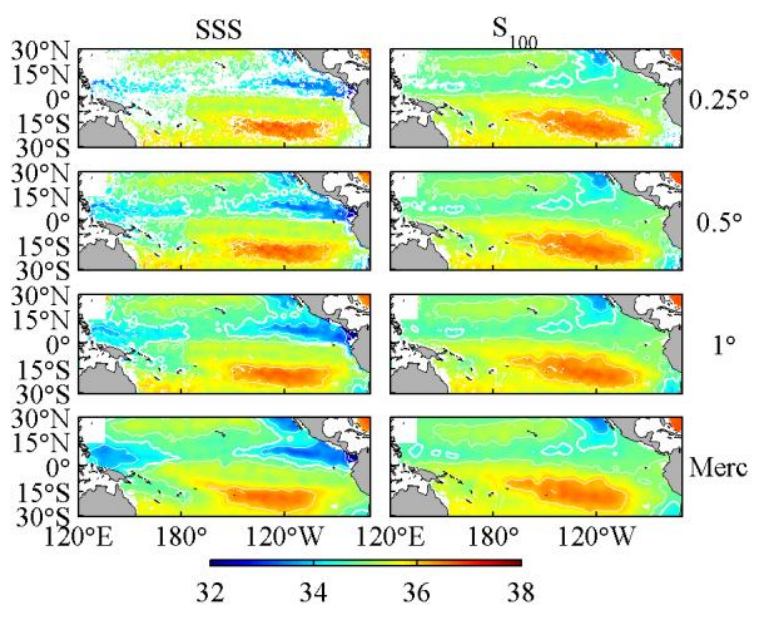

Fig. 1. SSS (left columns) and retrieved salinity at $100 \mathrm{~m}$ (right columns) using Method I (Eq. 2) and inputs from (from top to bottom) monthly SMOS SSS with $1 / 4^{\circ}, 1 / 2^{\circ}, 1^{\circ}$ resolution, and monthly ARIVO SSS on $1 / 2^{\circ}$ Mercator grids, for July 2011. Bold white lines are the 34.6 isohalines. 
(Method III), $\sigma_{2}$ and $\sigma_{3}$ are $\sim 10 \%$ less than $\sigma_{0}$. Below that, $\sigma_{2}$ and $\sigma_{3}$ are very close to $\sigma_{0}$. It suggests that the spatial resolution is an important factor for accuracy of the SSS inputs as well as of the retrieved $\mathrm{S}$ in the mixed layer; in contrast, the satellite SSS observations incline to play an insignificant role below the mixed layer. In additional tests (not shown) in which the remote SLA inputs were replaced with the in situ DHA inputs, the $\sigma_{2}$ patterns stayed roughly unchanged, implying that $\sigma_{2}-\sigma_{1}$ is mainly induced by $\sigma_{\mathrm{SSS}}$ rather than $\sigma_{\mathrm{SLA}}$ (Eq. 3 ).

A global performance description is shown in Fig. 3. On the whole, the SMSO SSS inputs perform in retrieving S increasingly better as its spatial resolution decreases; the temporal resolution is much less influential on S accuracy; the differences between high- and low- resolution maps gradually decrease as depth increases. At the surface and $50 \mathrm{~m}, \mathrm{p}_{2}$ is smaller than 1 only delimited in the tropical oceans. In detail, the SMSO $1 / 4^{\circ} \mathrm{SSS}$ input performs worse than climatology over most of the global oceans, in contrast to the $1^{\circ} \mathrm{SSS}$ input which performs better throughout the tropical oceans. At 150 $\mathrm{m}$, below the mixed layer, the regions where $\mathrm{p}_{2}<1$ expand to the extratropics, but excluding the southern oceans. At $300 \mathrm{~m}$, in the ocean interior, the regions with $\mathrm{p}_{2}>=1$ begin to prevail along the narrow equatorial bands. Below $400 \mathrm{~m}$, the patterns are gradually reshaped: the regions where $\mathrm{p}_{2}<1$ are concentrated around the northwestern Pacific Ocean, southwestern tropical Pacific Ocean, southern Indian Ocean, northern and southern Atlantic Ocean. For each map, $\mathrm{p}_{2}$ is always greater than 1 in regions south of $45^{\circ} \mathrm{S}$ and within the Arabian Sea and the Bengal Bay. For most areas, $\mathrm{p}_{2}$ stays above 0.8 , which indicates that the use of SMSO SSS inputs reduces the rms differences of $\mathrm{S}$ by less than $20 \%$. On the

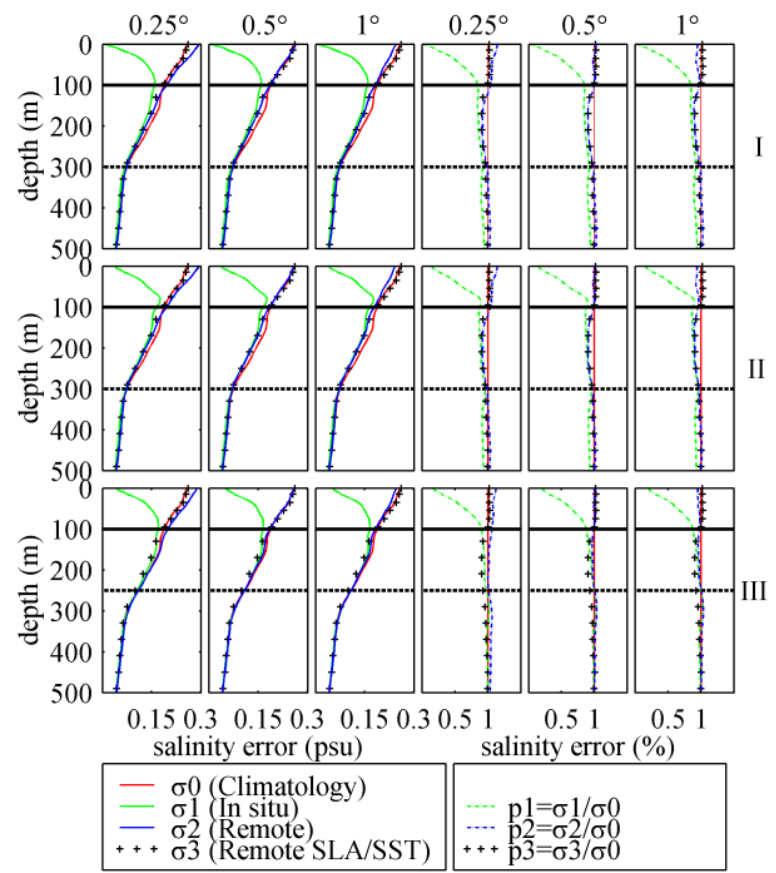

Fig. 2. Left three columns: profiles of $\sigma_{0}$ (solid red), $\sigma_{1}$ (solid green), $\sigma_{2}$ (solid blue), and $\sigma_{3}$ (black cross). Right three columns: profiles of $\mathrm{p}_{1}=\sigma_{1} / \sigma_{0}$ (dashed green), $\mathrm{p}_{2}=\sigma_{2} / \sigma_{0}$ (dashed blue), and $\mathrm{p}_{3}=\sigma_{3} / \sigma_{0}$ (black cross). The methods used are (from top to bottom) Method I, II and III. The SMOS SSS data used are 10 days composite with (first and fourth columns) $1 / 4^{\circ}$, (second and fifth columns) $1 / 2^{\circ}$, and (third and sixth columns) $1^{\circ}$ resolution. Up to 20046 independent S profiles in the tropical Pacific

Ocean $\left(120^{\circ} \mathrm{E} \sim 70^{\circ} \mathrm{W} ; 30^{\circ} \mathrm{S} \sim 30^{\circ} \mathrm{N}\right)$ for the year 2011 are used.

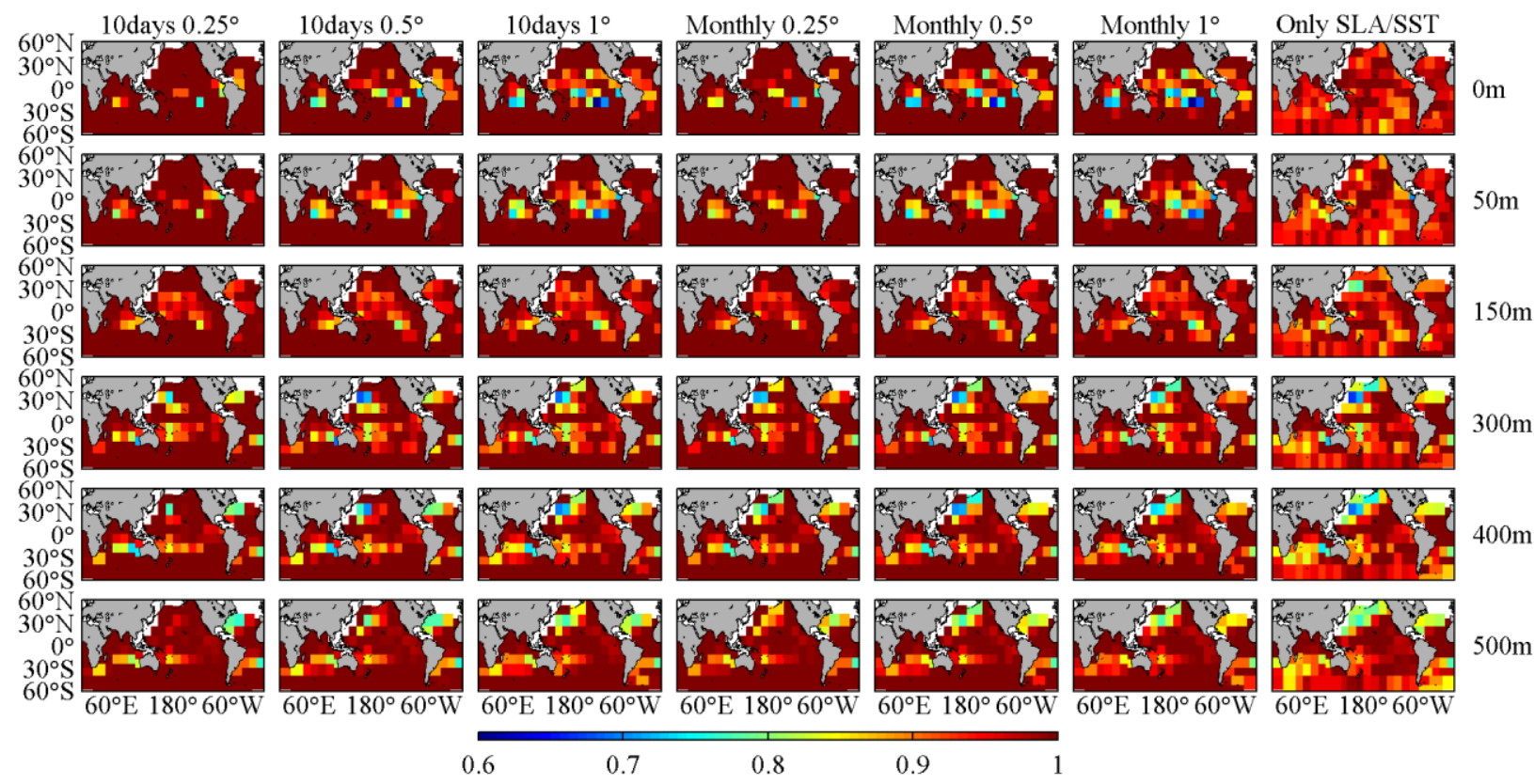

Fig. 3. The $\mathrm{p}_{2}=\sigma_{2} / \sigma_{0}$ (left six columns) and $\mathrm{p}_{3}=\sigma_{3} / \sigma_{0}$ (seventh column) at (from top to bottom) $0,50,150,300,400,500 \mathrm{~m}$. The SMOS SSS data used are (first to sixth columns) 10 days composite with $1 / 4^{\circ}, 1 / 2^{\circ}, 1^{\circ}$ resolution, and monthly composite with $1 / 4^{\circ}, 1 / 2^{\circ}, 1^{\circ}$ resolution. Up to 97475 independent $S$ profiles for the year 2011 are used. Values are computed in $15^{\circ} \times 15^{\circ}$ boxes and the $\mathrm{S}$ is averaged from the outputs of the three methods. 


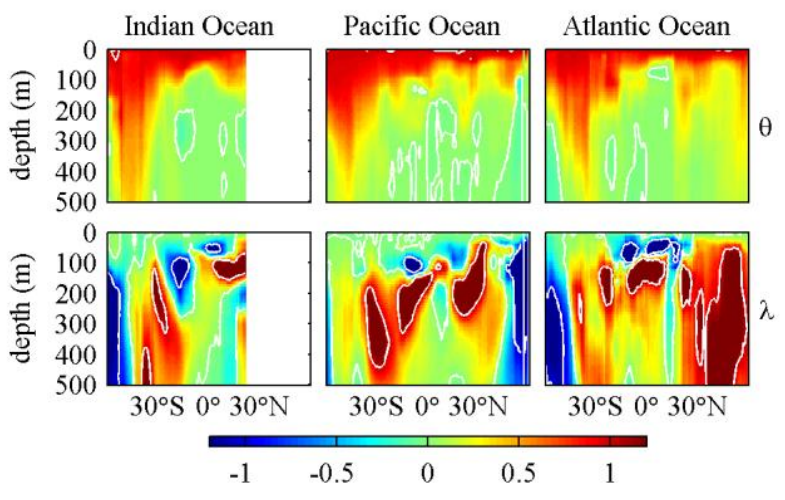

Fig. 4. Depth-latitude sections of $\theta$ and $\lambda$ along (from left to right) $70^{\circ} \mathrm{E}$ in the Indian Ocean, $160^{\circ} \mathrm{W}$ in the Pacific Ocean, $30^{\circ} \mathrm{W}$ in the Atlantic Ocean.

other hand, the distribution of $\mathrm{p}_{3}$ is uniform at all depths, and the corresponding reconstructed $\mathrm{S}$ is slightly better than the climatology on the global scale. In the mixed layer, $\mathrm{p}_{2}$ is slightly smaller than $\mathrm{p}_{3}$ in the tropical oceans; below that, $\mathrm{p}_{2}$ is distributed basically consistent with $\mathrm{p}_{3}$, except, again, in the areas south of $45^{\circ} \mathrm{S}$.

Figure 4 conclusively illustrates the way the SSS errors are projected at depth (see Eq. 3). In the top mixed layer, the $\theta$ has uniformly high values at all latitudes. This explains why in that layer $\sigma_{2}$ varies sensitively with the SSS resolution (Fig. 2), and $\mathrm{p}_{2}$ keeps the surface structures (Fig. 3). Below $100 \mathrm{~m}$, most $\theta$ values are close to zero, except that a vertical tongue of maximum $\theta$ value reaches down to $400 \sim 500 \mathrm{~m}$ in the southern oceans, indicating that the SSS errors there can stay influential at depth. This accounts for the gradually disappearing surface $\mathrm{p}_{2}$ patterns below $150 \mathrm{~m}$, and the discrepancy between $\mathrm{p}_{2}$ and $\mathrm{p}_{3}$ patterns in deep oceans south of $45^{\circ} \mathrm{S}$, where large SMOS SSS errors offset the influence of SLA (Fig. 3). As regard $\lambda$, the tropics are dominated by negative values from surface down to $\sim 100 \mathrm{~m}$, then positive values downwards. The maximum positive values are concentrated between $100 \sim 300$ $\mathrm{m}$, which is consistent with the $\sigma_{2}$ and $\sigma_{3}$ profiles, robustly smaller than $\sigma_{0}$, in that layer (Fig. 2). At higher latitudes, the northern Atlantic and Pacific Ocean show striking negative and positive tongues respectively, and the southern Indian and Atlantic Ocean each shows a clear negative tongue from nearsurface to depths. It reminds of the displacement of small $\mathrm{p}_{2}$ to mid-high-latitudes below $150 \mathrm{~m}$ (Fig. 3).

\section{DISCUSSION AND CONCLUSION}

The SMOS SSS inputs have been evaluated in retrieving $S$ profiles and proved to work differently at different depths and regions. In the mixed layer, the retrieved $S$ is mainly influenced by the SSS information. The higher the SSS-S regression coefficients, the more errors in S projected from the SSS. The spatial resolution confirmed to be an important factor for the $S$ retrieval. The SMOS SSS inputs perform better than, equivalent to, or worse than the climatology, with a resolution of $1^{\circ}, 1 / 2^{\circ}, 1 / 4^{\circ}$, respectively, in the tropics, and always worse elsewhere. This is consistent with Jordà and Gomis [6] who concluded that "the greatest error reductions come from the increase in the spatial-data coverage, even if the temporal coverage is scarce". Besides, the projection generally reduces the mesoscale structures in the $1 / 4^{\circ} \mathrm{SSS}$ fields. Below that layer, the retrieved $S$ is mainly influenced by the SLA information. The higher the SLA-S regression coefficients, the higher the S accuracy benefiting from the SLA. The SSS-S regression coefficients, by contrast, are negligible, leading to the disappeared surface error patterns. An exception is the southern oceans where the maximum SSS-S regression coefficients propagate the SSS errors down to $400 \sim 500 \mathrm{~m}$.

Up to now, the SMOS SSS measurements have not greatly improved the estimates of vertical salinity structures, especially in the extratropics and with "eddy permitting" resolution. For this reason, more accurate satellite SSS measurements are needed and currently under development.

\section{ACKNOWLEDGMENT}

The authors would like to thank the Coriolis data center for the CORA3 and ARIVO datasets, the Ocean Salinity Expertise Center (CECOS) for the SMOS SSS data, the National Climatic Data Center at NOAA for the SST data, and the Ssalto/Duacs Center for the altimeter data.

\section{REFERENCES}

[1] Y. H. Kerr, P. Waldteufel, J.-P. Wigneron, J. Martinuzzi, J. Font, and M. Berger, "Soil moisture retrieval from space: The Soil Moisture and Ocean Salinity (SMOS) mission," IEEE Trans. Geosci. Remote Sens., vol. 39, pp. 1729-1735, 2001.

[2] B. B. Nardelli and R. Santoleri, "Methods for the reconstruction of vertical profiles from surface data: Multivariate analyses, residual GEM, and variable temporal signals in the North Pacific Ocean," J. Atmos. Ocean. Technol., vol. 22, pp. 17621781, 2005.

[3] N. Agarwal, R. Sharma, S. Basu, and V. K. Agarwal, "Derivation of salinity profiles in the Indian ocean from satellite surface observations," IEEE Geosci. Remote Sens. Lett., vol. 4, pp. 322-325, 2007.

[4] X. Wu, X.-H. Yan, Y.-H. Jo, and W. Timothy Liu, "Estimation of Subsurface Temperature Anomaly in the North Atlantic using a Self-Organizing Map Neural Network," J. Atmos. Ocean. Technol., vol. 29, pp. 1675-1688, 2012.

[5] B. Buongiorno Nardelli, S. Guinehut, A. Pascual, Y. Drillet, S. Ruiz, and S. Mulet, "Towards high resolution mapping of 3-D mesoscale dynamics from observations," Ocean Sci., vol. 8, pp. 885-901, 2012.

[6] G. Jordà and D. Gomis, "Toward SMOS L4 SSS products: Improving L3 SSS with auxiliary SSS data," IEEE Trans. Geosci. Remote Sens., vol. 48, pp. 2204-2214, 2010.

[7] S. Guinehut, P.-Y. Le Traon, and G. Larnicol, "What can we learn from Global Altimetry/Hydrography comparisons?," Geophys. Res. Lett., vol. 33, p. L10604, 2006.

[8] S. Guinehut, P. Le Traon, G. Larnicol, and S. Philipps, "Combining Argo and remote-sensing data to estimate the ocean three-dimensional temperature fields-A first approach based on simulated observations," J. Mar. Syst., vol. 46, pp. 85-98, 2004.

[9] M. R. Carnes, W. J. Teague, and J. L. Mitchell, "Inference of subsurface thermohaline structure from fields measurable by satellite," J. Atmos. Ocean. Technol., vol. 11, p. 551, 1994. 